This is an electronic reprint of the original article. This reprint may differ from the original in pagination and typographic detail.

\author{
Author(s): Mason, P. J. R.; Simpson, J.; Paul, E. S.; Riley, M. A.; Auranen, Kalle; Badran, Hussam; \\ Baron, J. S.; Boston, A. J.; Davis-Merry, T.; Grahn, Tuomas; Greenlees, Paul; Herzan, \\ Andrej; Jakobsson, Ulrika; Joss, D. T.; Julin, Rauno; Juutinen, Sakari; Konki, Joonas; \\ Leino, Matti; McPeake, C.; Miller, S. L.; O'Neill, G.; Pakarinen, Janne; Papadakis, \\ Philippos; Partanen, Jari; Peura, Pauli; Rahkila, Panu; Ruotsalainen, Panu; Sandzelius,
}

Title: $\quad$ Spectroscopy of $161 \mathrm{Hf}$ from low to high spin

Year: $\quad 2014$

Version:

Please cite the original version:

Mason, P. J. R., Simpson, J., Paul, E. S., Riley, M. A., Auranen, K., Badran, H., Baron, J. S., Boston, A. J., Davis-Merry, T., Grahn, T., Greenlees, P., Herzan, A., Jakobsson, U., Joss, D. T., Julin, R., Juutinen, S., Konki, J., Leino, M., McPeake, C., . . Wrigh, J. P. (2014). Spectroscopy of $161 \mathrm{Hf}$ from low to high spin. Physical Review C, 90(5), Article 054331. https://doi.org/10.1103/PhysRevC.90.054331

All material supplied via JYX is protected by copyright and other intellectual property rights, and duplication or sale of all or part of any of the repository collections is not permitted, except that material may be duplicated by you for your research use or educational purposes in electronic or print form. You must obtain permission for any other use. Electronic or print copies may not be offered, whether for sale or otherwise to anyone who is not an authorised user. 


\title{
Spectroscopy of ${ }^{161} \mathrm{Hf}$ from low to high spin
}

\author{
P. J. R. Mason, ${ }^{1}$ J. Simpson, ${ }^{1, *}$ E. S. Paul, ${ }^{2}$ M. A. Riley, ${ }^{3}$ K. Auranen,${ }^{4}$ H. Badran, ${ }^{4}$ J. S. Baron, ${ }^{3}$ A. J. Boston, ${ }^{2}$ \\ T. Davis-Merry, ${ }^{2}$ T. Grahn, ${ }^{4}$ P. T. Greenlees, ${ }^{4}$ A. Herzáň, ${ }^{4}$ U. Jakobsson, ${ }^{4}$ D. T. Joss, ${ }^{2}$ R. Julin, ${ }^{4}$ S. Juutinen, ${ }^{4}$ J. Konki, ${ }^{4}$ \\ M. Leino, ${ }^{4}$ C. McPeake, ${ }^{2}$ S. L. Miller, ${ }^{3}$ G. O’Neill, ${ }^{2}$ J. Pakarinen, ${ }^{4}$ P. Papadakis, ${ }^{4}$ J. Partanen, ${ }^{4}$ P. Peura,${ }^{4}$ P. Rahkila, ${ }^{4}$ \\ P. Ruotsalainen, ${ }^{4}$ M. Sandzelius, ${ }^{4}$ J. Sarén, ${ }^{4}$ C. Scholey, ${ }^{4}$ J. F. Sharpey-Schafer, ${ }^{5}$ L. Sinclair, ${ }^{6}$ J. Sorri, ${ }^{4}$ S. Stolze, ${ }^{4}$ J. Uusitalo, ${ }^{4}$ \\ K. Villafani, ${ }^{3}$ F. Wearing, ${ }^{2}$ and J. P. Wright ${ }^{2}$ \\ ${ }^{1}$ STFC Daresbury Laboratory, Daresbury, Warrington, WA4 4AD, United Kingdom \\ ${ }^{2}$ Oliver Lodge Laboratory, University of Liverpool, Liverpool, L69 7ZE, United Kingdom \\ ${ }^{3}$ Department of Physics, Florida State University, Tallahassee, Florida 32306, USA \\ ${ }^{4}$ Department of Physics, University of Jyväskylä, FI-40014 Jyväskylä, Finland \\ ${ }^{5}$ iThemba Laboratory, P.O. Box 722, Somerset West 7129, South Africa \\ ${ }^{6}$ Department of Physics, University of York, York, YO10 5DD, United Kingdom
}

(Received 26 September 2014; published 21 November 2014)

\begin{abstract}
Excited states in the neutron-deficient nucleus ${ }_{72}^{161} \mathrm{Hf}_{89}$ have been populated using the ${ }^{118} \mathrm{Sn}\left({ }^{48} \mathrm{Ti}, 5 n\right){ }^{161} \mathrm{Hf}$ and ${ }^{110} \mathrm{Pd}\left({ }^{56} \mathrm{Fe}, 5 n\right){ }^{161} \mathrm{Hf}$ fusion-evaporation reactions at 240 and $270 \mathrm{MeV}$, respectively. The level scheme for ${ }^{161} \mathrm{Hf}$ has been extended with the observation of new band structures and an $I^{\pi}=\left(13 / 2^{+}\right)$isomeric state with a half-life of $4.8(2) \mu$ s has been identified. The decay path from this isomer to the $\left(7 / 2^{-}\right)$ground state is established. The yrast band, based on the $\left(13 / 2^{+}\right)$isomeric state, is extended up to $\left(73 / 2^{+}\right)$and side band structures are identified up to $\left(69 / 2^{-}\right)$and $\left(55 / 2^{-}\right)$. Quasiparticle assignments for these rotational structures are made on the basis of their alignment properties and Woods-Saxon cranked shell-model calculations.
\end{abstract}

DOI: 10.1103/PhysRevC.90.054331

PACS number(s): 27.70.+q, 21.10.Re, 23.20.Lv, 25.70.Gh

\section{INTRODUCTION}

Neutron-deficient rare-earth nuclei with $A \sim 160$ and $N=$ 88-92 have become classic cases for the study of normaldeformed nuclear states at high angular momentum. These studies have revealed much information about the structure of nuclei, in particular, the mapping out of the nuclear shape with increasing angular momentum. At spins up to around $30 \hbar-40 \hbar$ these nuclei are dominated by collective structures that undergo alignments of both neutron and proton pairs as the rotational frequency is increased. The observation of the associated band crossings has provided detailed information concerning the configuration assignments. In the $N=89$ isotones the alignment in the yrast structure, based on a neutron $i_{13 / 2}$ configuration, has been observed to evolve as a function of proton number. In the deformed ${ }^{157} \mathrm{Er}$ [1] and ${ }^{159} \mathrm{Yb}$ [2] nuclei, the first crossing is a combination of the alignment of the second pair of neutron $i_{13 / 2}$ particles and the first proton $h_{11 / 2}$ particles. In the much less deformed nucleus ${ }^{163} \mathrm{~W}$ [3] the neutron $h_{9 / 2}$ and $f_{7 / 2}$ orbitals are low in energy and cross the yrast structure well before the neutron $i_{13 / 2}$ crossing. In this work the nucleus ${ }^{161} \mathrm{Hf}$ is observed to high spin to investigate the nature of the alignments in a nucleus that is predicted to have an intermediate deformation between these neighboring isotones.

In a previous study the yrast band of this nucleus was established to $I^{\pi}=\left(49 / 2^{+}\right)$(with a tentative extension to $53 / 2^{+}$) [4] with a side structure comprising three transitions that extend up to $I^{\pi}=\left(43 / 2^{-}\right)$. This paper further extends these structures to higher spins and establishes a new side

*Corresponding author: john.simpson@stfc.ac.uk band structure. The rotational bands observed are compared with the predictions of cranked shell-model calculations and configurations are assigned. This work also establishes that the bandhead of the yrast band is isomeric and has $I^{\pi}=\left(13 / 2^{+}\right)$, and we determine its decay path to the $\left(7 / 2^{-}\right)$ground state.

\section{EXPERIMENT AND DATA ANALYSIS}

Excited states in ${ }^{161} \mathrm{Hf}$ were populated in fusionevaporation reactions at the Accelerator Laboratory of the University of Jyväskylä, Finland, with two different combinations of beam and target nuclei. The first reaction was ${ }^{118} \mathrm{Sn}\left({ }^{48} \mathrm{Ti}, 5 n\right){ }^{161} \mathrm{Hf}$ with a beam energy of $240 \mathrm{MeV}$. Stacked ${ }^{118} \mathrm{Sn}$ targets with total thicknesses of $\sim 1.2 \mathrm{mg} / \mathrm{cm}^{2}$ were used. The second reaction used was ${ }^{110} \mathrm{Pd}\left({ }^{56} \mathrm{Fe}, 5 n\right){ }^{161} \mathrm{Hf}$ with a beam energy of $270 \mathrm{MeV}$. This reaction produces the same ${ }^{166} \mathrm{Hf}$ compound nucleus as the ${ }^{118} \mathrm{Sn}\left({ }^{48} \mathrm{Ti}, 5 n\right){ }^{161} \mathrm{Hf}$ reaction and was adopted because of the more durable target material in beam. Two stacked ${ }^{110} \mathrm{Pd}$ targets were used with a total thickness of $\sim 1.2 \mathrm{mg} / \mathrm{cm}^{2}$. The ${ }^{118} \mathrm{Sn}\left({ }^{48} \mathrm{Ti}, 5 n\right){ }^{161} \mathrm{Hf}$ and ${ }^{110} \mathrm{Pd}\left({ }^{56} \mathrm{Fe}, 5 n\right){ }^{161} \mathrm{Hf}$ reactions were run for $\sim 39$ and $\sim 82 \mathrm{~h}$, respectively, with average beam intensities of $\sim 5$ particle-nA and $\sim 8$ particle-nA, respectively.

Promptly emitted $\gamma$ rays from the reaction products were detected in the JUROGAM II array, consisting of 24 Comptonsuppressed clover and 13 tapered HPGe detectors surrounding the target chamber [5]. Recoiling reaction products were separated from the primary beam in the RITU gas-filled separator [6] and transported to the focal plane, where they were implanted into the double-sided silicon strip detectors (DSSDs) of the GREAT spectrometer [7]. Energy-loss and time-of-flight measurements in GREAT were used to distinguish the reaction products from any scattered beam 
transported through RITU. $\gamma$-ray transitions occurring after implantation at the focal plane were detected with three clover HPGe detectors and the GREAT planar HPGe detector.

Data were recorded using the triggerless total data readout (TDR) data-acquisition system [8], where events in each detector are recorded individually and time-stamped using a $100-\mathrm{MHz}$ clock. The data from both reactions were sorted into a series of two- and three-dimensional histograms (matrices and cubes) using the GRAIN software package [9]. Matrices and cubes were analyzed with the RADWARE software suite [10].

The recoil-isomer tagging technique [11], in which prompt $\gamma$ rays detected at the target position are correlated to delayed $\gamma$ rays detected at the focal plane, was utilized in the analysis of the data. By correlating prompt $\gamma$ rays to the decay of an isomeric state, transitions above the isomer can be selected, creating spectra that are mostly free from contamination by other reaction products. Prompt spectra and matrices correlated with decays from an isomer will henceforth be termed isomer gated. In ${ }^{161} \mathrm{Hf}$ isomer-gated histograms, only delayed $\gamma$ rays detected within $0-5 \mu$ s of an implantation at the focal plane were considered, to minimize the background of $\gamma$ rays resulting from longer-lived $\beta$ decays as well as decays from subsequent recoil implantations. A total of $1.8 \times 10^{10}$ three-fold $\left(\gamma^{3}\right)$ events in the JUROGAM II array were unpacked from the dataset and used in the analysis.

\section{RESULTS}

Figure 1 shows spectra of prompt and delayed $\gamma$ rays identified in this work as belonging to ${ }^{161} \mathrm{Hf}$. The level scheme for ${ }^{161} \mathrm{Hf}$ deduced in this work is shown in Fig. 2 while Table I lists the measured $\gamma$-ray properties.

The half-life of the isomeric state was determined by fitting an exponential decay curve to the distributions of time differences between implantation of a recoil at the focal plane

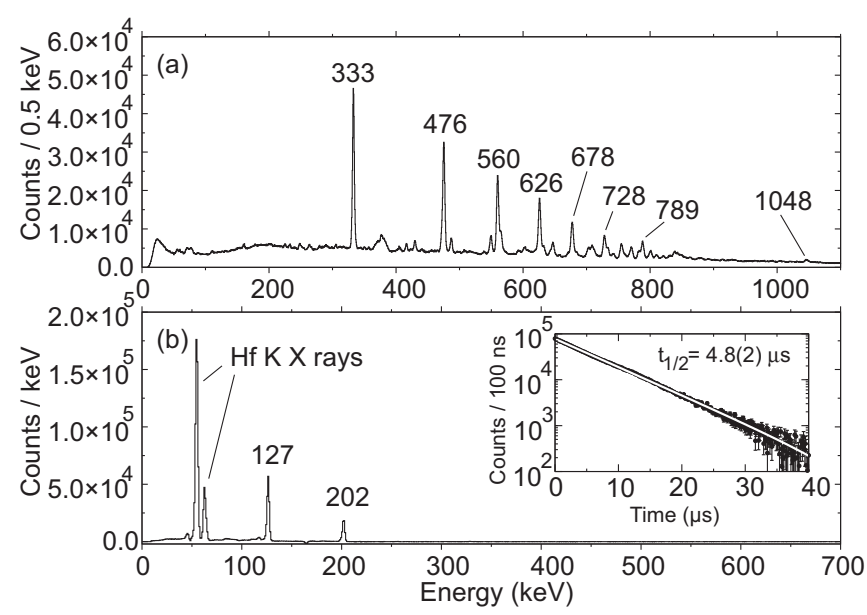

FIG. 1. (a) Spectrum showing the prompt $\gamma$ rays correlated with the 127- and 202-keV transitions below the ${ }^{161} \mathrm{Hf}$ isomer. (b) Delayed $\gamma$ rays correlated with the $476-\mathrm{keV}$ prompt transition in ${ }^{161} \mathrm{Hf}$. The inset to panel (b) shows a time difference spectrum between recoil implantation at the focal plane and detection of the 202-keV $\gamma$ ray in the GREAT planar detector. The solid line is an exponential decay fitted to the data.

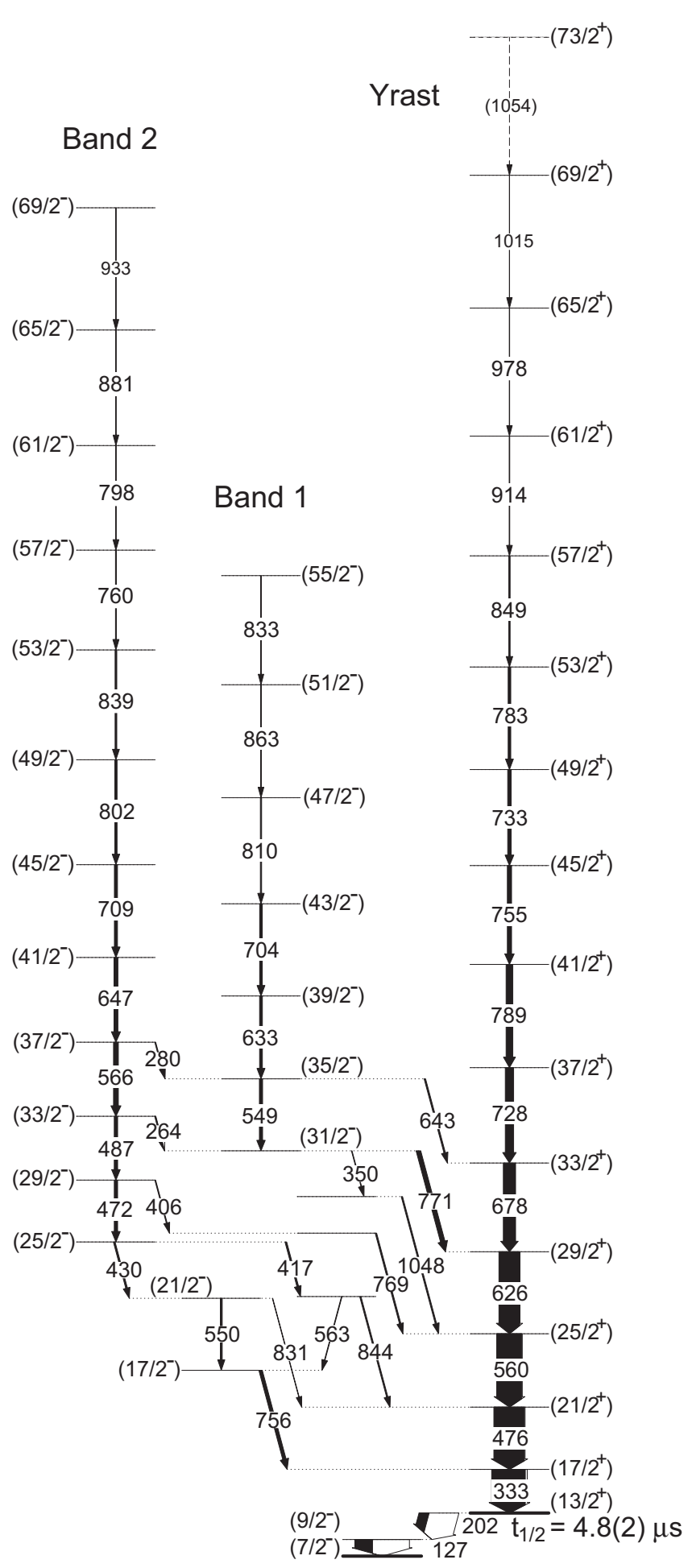

FIG. 2. Level scheme for ${ }^{161} \mathrm{Hf}$ deduced from this study. The widths of the arrows are proportional to the intensities of the $\gamma$-ray transitions and the white part of the arrows represents the calculated internal conversion component.

and detection of the 202- and $127-\mathrm{keV} \gamma$ rays. A value of $t_{1 / 2}=4.8(2) \mu \mathrm{s}$ was obtained for the half-life. The inset to Fig. 1(b) shows an example time spectrum for the decay of the $202-\mathrm{keV}$ transition. Two mutually coincident $\gamma$-ray transitions of 202 and $127 \mathrm{keV}$ were identified following the decay of 
TABLE I. Measured $\gamma$-ray energies, intensities, and initial and final spins for ${ }^{161} \mathrm{Hf}$ from this work. The intensities of the prompt and delayed transitions are normalized separately.

\begin{tabular}{|c|c|c|}
\hline$E_{\gamma}(\mathrm{keV})$ & $I_{\gamma}$ & $I_{i}^{\pi} \rightarrow I_{f}^{\pi}$ \\
\hline $126.8(3)$ & $100(2)$ & $\left(9 / 2^{-} \rightarrow 7 / 2^{-}\right)$ \\
\hline 202.2(4) & $56(3)$ & $\left(13 / 2^{+} \rightarrow 9 / 2^{-}\right)$ \\
\hline 263.7(10) & $3(1)$ & $\left(33 / 2^{-} \rightarrow 31 / 2^{-}\right)$ \\
\hline $279.6(14)$ & 2(1) & $\left(37 / 2^{-} \rightarrow 35 / 2^{-}\right)$ \\
\hline $333.3(4)$ & $100(1)$ & $\left(17 / 2^{+} \rightarrow 13 / 2^{+}\right)$ \\
\hline $349.8(22)$ & $2(2)$ & $\left(31 / 2^{-}\right) \rightarrow$ \\
\hline $405.5(14)$ & $2(1)$ & $\left(29 / 2^{-}\right) \rightarrow$ \\
\hline $416.7(8)$ & $5(1)$ & $\left(25 / 2^{-}\right) \rightarrow$ \\
\hline $430.2(7)$ & $5(1)$ & $\left(25 / 2^{-} \rightarrow 21 / 2^{-}\right)$ \\
\hline $472.4(7)$ & $10(3)$ & $\left(29 / 2^{-} \rightarrow 25 / 2^{-}\right)$ \\
\hline $475.5(5)$ & $91(3)$ & $\left(21 / 2^{+} \rightarrow 17 / 2^{+}\right)$ \\
\hline $487.4(5)$ & $10(1)$ & $\left(33 / 2^{-} \rightarrow 29 / 2^{-}\right)$ \\
\hline $549.4(7)$ & $9(1)$ & $\left(35 / 2^{-} \rightarrow 31 / 2^{-}\right)$ \\
\hline $549.9(9)$ & $6(1)$ & $\left(21 / 2^{-} \rightarrow 17 / 2^{-}\right)$ \\
\hline $560.3(5)$ & $75(2)$ & $\left(25 / 2^{+} \rightarrow 21 / 2^{+}\right)$ \\
\hline $563.3(12)$ & $2(1)$ & $\rightarrow\left(17 / 2^{-}\right)$ \\
\hline $565.7(6)$ & $14(1)$ & $\left(37 / 2^{-} \rightarrow 33 / 2^{-}\right)$ \\
\hline $626.4(5)$ & $62(2)$ & $\left(29 / 2^{+} \rightarrow 25 / 2^{+}\right)$ \\
\hline $632.8(8)$ & $8(1)$ & $\left(39 / 2^{-} \rightarrow 35 / 2^{-}\right)$ \\
\hline 643.3(9) & $4(1)$ & $\left(35 / 2^{-} \rightarrow 33 / 2^{+}\right)$ \\
\hline $647.2(6)$ & $12(1)$ & $\left(41 / 2^{-} \rightarrow 37 / 2^{-}\right)$ \\
\hline $677.7(5)$ & $36(2)$ & $\left(33 / 2^{+} \rightarrow 29 / 2^{+}\right)$ \\
\hline 704.0(9) & $8(1)$ & $\left(43 / 2^{-} \rightarrow 39 / 2^{-}\right)$ \\
\hline $708.7(7)$ & $9(1)$ & $\left(45 / 2^{-} \rightarrow 41 / 2^{-}\right)$ \\
\hline $728.4(5)$ & $25(1)$ & $\left(37 / 2^{+} \rightarrow 33 / 2^{+}\right)$ \\
\hline $733.3(6)$ & $10(1)$ & $\left(49 / 2^{+} \rightarrow 45 / 2^{+}\right)$ \\
\hline $754.7(6)$ & $12(2)$ & $\left(45 / 2^{+} \rightarrow 41 / 2^{+}\right)$ \\
\hline $756.5(10)$ & $10(2)$ & $\left(17 / 2^{-} \rightarrow 17 / 2^{+}\right)$ \\
\hline $760.4(13)$ & $3(1)$ & $\left(57 / 2^{-} \rightarrow 53 / 2^{-}\right)$ \\
\hline $768.5(13)$ & $4(1)$ & $\rightarrow\left(25 / 2^{+}\right)$ \\
\hline 771.1(7) & $13(1)$ & $\left(31 / 2^{-} \rightarrow 29 / 2^{+}\right)$ \\
\hline $783.3(8)$ & $8(1)$ & $\left(53 / 2^{+} \rightarrow 49 / 2^{+}\right)$ \\
\hline $788.5(5)$ & $20(1)$ & $\left(41 / 2^{+} \rightarrow 37 / 2^{+}\right)$ \\
\hline $798.5(14)$ & $2(1)$ & $\left(61 / 2^{-} \rightarrow 57 / 2^{-}\right)$ \\
\hline $801.6(8)$ & $7(1)$ & $\left(49 / 2^{-} \rightarrow 45 / 2^{-}\right)$ \\
\hline $810.0(14)$ & $4(1)$ & $\left(47 / 2^{-} \rightarrow 43 / 2^{-}\right)$ \\
\hline $831.1(15)$ & $2(1)$ & $\left(21 / 2^{-} \rightarrow 21 / 2^{+}\right)$ \\
\hline $833.0(13)$ & $1(1)$ & $\left(55 / 2^{-} \rightarrow 51 / 2^{-}\right)$ \\
\hline $838.8(9)$ & $5(1)$ & $\left(53 / 2^{-} \rightarrow 49 / 2^{-}\right)$ \\
\hline $844.4(6)$ & $4(1)$ & $\rightarrow\left(21 / 2^{+}\right)$ \\
\hline $848.9(12)$ & $4(1)$ & $\left(57 / 2^{+} \rightarrow 53 / 2^{+}\right)$ \\
\hline $863(3)$ & $2(1)$ & $\left(51 / 2^{-} \rightarrow 47 / 2^{-}\right)$ \\
\hline $881.4(12)$ & $1(1)$ & $\left(65 / 2^{-} \rightarrow 61 / 2^{-}\right)$ \\
\hline $914.3(20)$ & $2(1)$ & $\left(61 / 2^{+} \rightarrow 57 / 2^{+}\right)$ \\
\hline $933(2)$ & $1(1)$ & $\left(69 / 2^{-} \rightarrow 65 / 2^{-}\right)$ \\
\hline $978(2)$ & $1(1)$ & $\left(65 / 2^{+} \rightarrow 61 / 2^{+}\right)$ \\
\hline $1015(2)$ & $1(1)$ & $\left(69 / 2^{+} \rightarrow 65 / 2^{+}\right)$ \\
\hline $1048(3)$ & $4(1)$ & $\rightarrow\left(25 / 2^{+}\right)$ \\
\hline $1053(2)$ & $1(1)$ & $\left(73 / 2^{+} \rightarrow 69 / 2^{+}\right)$ \\
\hline
\end{tabular}

the isomer in ${ }^{161} \mathrm{Hf}$. The multipolarities of these transitions were deduced through measurement of their K-shell internal conversion coefficients, $\alpha_{K}$. The conversion coefficients were measured by comparing the intensities of the $\gamma$ rays and $\mathrm{Hf}$ $\mathrm{K}$-shell $\mathrm{X}$ rays in a matrix of delayed $\gamma$ rays detected within

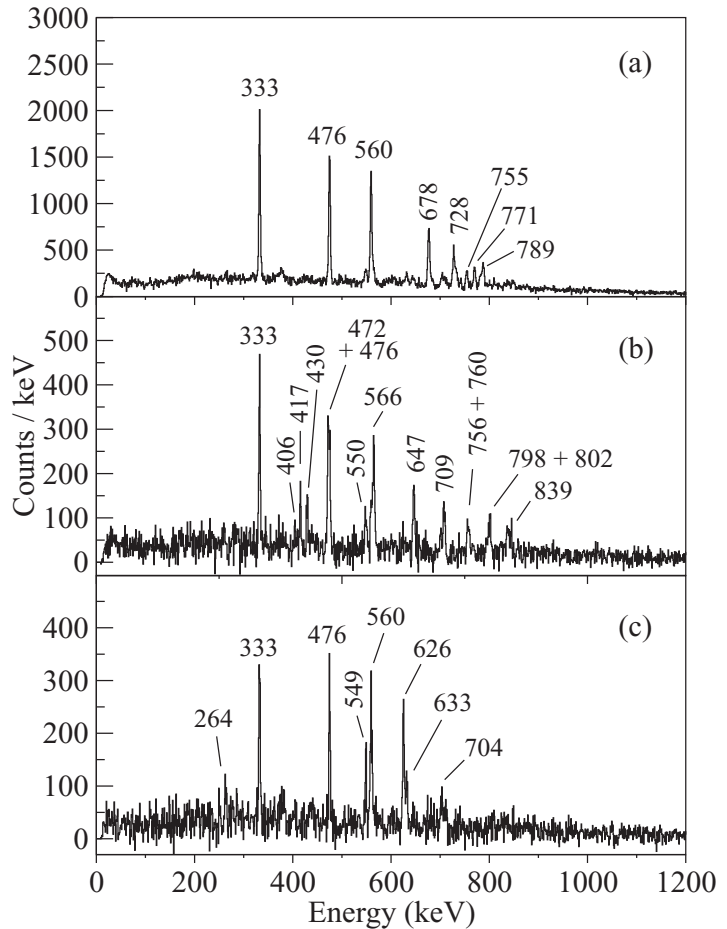

FIG. 3. Spectra of prompt $\gamma$ rays in coincidence with the (a) 626-, (b) 487-, and (c) 771-keV transitions in the ${ }^{161} \mathrm{Hf}$ isomer-gated matrix.

$5 \mu$ s of a recoil implant at the focal plane. Coincidence gates were set on the delayed $\gamma$ rays in ${ }^{161} \mathrm{Hf}$ to ensure only $\mathrm{X}$ rays associated with conversion of the transition of interest were measured. Coefficients of $\alpha_{\mathrm{K}}=2.8(4)$ and $\alpha_{\mathrm{K}}=1.7(2)$ were found for the 202- and $127-\mathrm{keV}$ transitions, respectively. The relatively large error in these measurements is principally due to uncertainty in the efficiency of the GREAT planar detector. The coefficients are consistent with $M 2$ and $M 1$ multipolarities for the 202- and $127-\mathrm{keV}$ transitions, respectively, using the BrIcc internal conversion coefficient database [12].

The prompt level scheme was constructed by using information from a ${ }^{161} \mathrm{Hf}$ isomer-gated $\gamma \gamma$ matrix and a nongated $\gamma^{3}$ cube. The yrast band has been extended to a spin of $\left(69 / 2^{+}\right)$and tentatively $\left(73 / 2^{+}\right)$. Our data are consistent with the scheme of Hübel et al. [4] up to $\left(49 / 2^{+}\right)$but the energy of the $735-\mathrm{keV}$ transition reported by Hübel et al. differs slightly from the $733 \mathrm{keV}$ observed in the present work. The 743-keV transition tentatively proposed by Hübel et al. placed above this was not confirmed in the present work.

Two side bands, labeled Bands 1 and 2, have also been observed in this work. Band 1 is consistent with the side band observed by Hübel et al., linked via a 771-keV transition to the yrast band. The band has been extended to a spin of $\left(55 / 2^{-}\right)$ and further linking transitions have been found. Band 2 is newly identified in this work and is linked to Band 1 and through intermediate states to the yrast band. Figure 3 and Fig. 4 show spectra from the isomer-gated $\gamma \gamma$ matrix and $\gamma^{3}$ cube, respectively, showing transitions in the yrast band, Band 1 , and Band 2.

Angular distributions were measured for the strongest prompt $\gamma$ rays by measuring their intensity as a function 


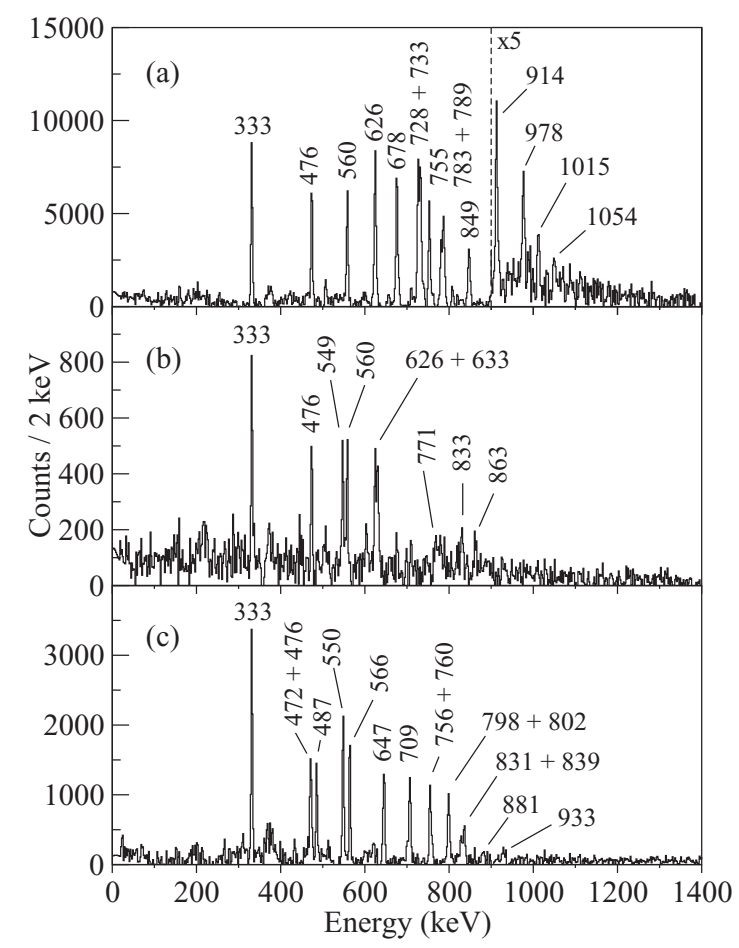

FIG. 4. Doubled-gated $\gamma$-ray spectra from the $\gamma^{3}$ cube showing the bands in ${ }^{161} \mathrm{Hf}$. (a) Spectrum showing $\gamma$ rays in the yrast band from gating a sum of 333-, 476-, and 560-keV peaks on the first axis and a sum of 849-, 914-, 978-, and 1015-keV peaks on the second axis of the cube. Above $900 \mathrm{keV}$, the spectrum is multiplied by a factor of 5 to clearly show the highest-spin transitions in the band. (b) Spectrum showing $\gamma$ rays in Band 1 from a double gate on the 704- and 810-keV transitions. (c) Spectrum showing $\gamma$ rays in Band 2 from gating a sum of the 472- and 487-keV gates on the first axis and the $430-\mathrm{keV}$ peak on the second axis of the $\gamma^{3}$ cube.

of detector angle in an isomer-gated spectrum. These distributions were compared with predicted distributions for pure stretched dipole and quadrupole transitions to estimate the spin change associated with the transitions. A Gaussian substate distribution with $\sigma / I=0.3$ [13] was assumed for the theoretical distributions. The data were corrected for efficiency and normalized to the distribution of the $333-\mathrm{keV}$ transition, which is assumed to be a pure stretched quadrupole. Sample distributions are shown in Fig. 5.

A quadrupole nature was deduced for the 487-, 560-, 566-, 626-, and 678-keV transitions, which are assumed to have stretched $E 2$ multipolarity. The $472-/ 476-\mathrm{keV}$ and 549/550-keV doublets also have combined distributions which are consistent with all of these transitions being stretched quadrupoles. The intensity of the $769-/ 771-\mathrm{keV}$ doublet is dominated by the $771-\mathrm{keV}$ transition and the distribution is consistent with that of a stretched dipole. Large errors due to poor statistics were found for the $430-\mathrm{keV}$ transition, making it difficult to determine a multipolarity, but its distribution is more consistent with that of a stretched quadrupole.

Due to the level of statistics available and contamination (in non-isomer-gated matrices) from other reaction products it

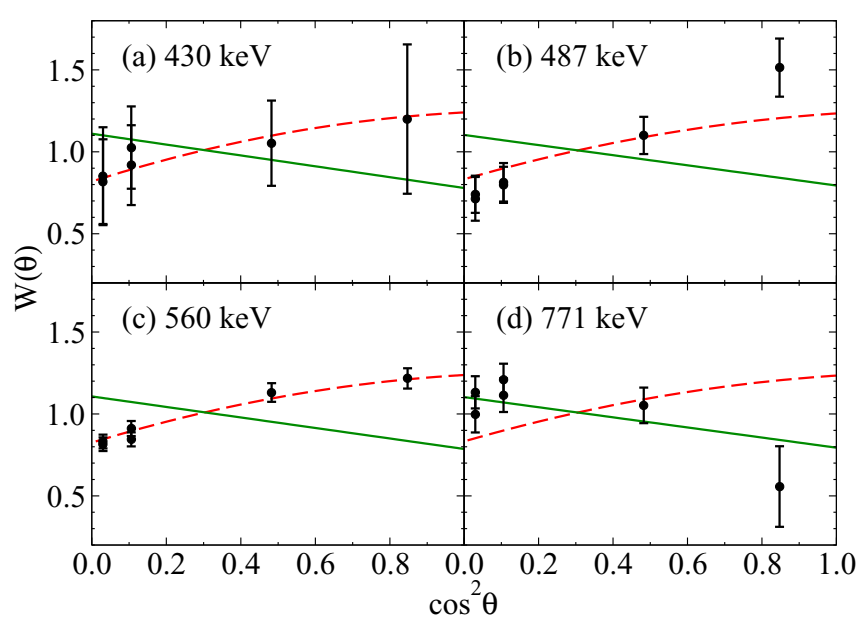

FIG. 5. (Color online) Angular distributions measured for the (a) 430-, (b) 487-, (c) 560-, and (d) 771-keV transitions. The solid and dashed lines show the theoretical distributions for pure stretched dipole and quadrupole transitions, respectively.

was not possible to produce gated angular correlations in this work.

\section{DISCUSSION}

\section{A. Quasiparticle alignments}

The structure of the bands in ${ }^{161} \mathrm{Hf}$ is interpreted in terms of quasiparticle configurations within the framework of Woods-Saxon cranking calculations using universal parameters $[14,15]$. Quasiparticle Routhians, $e^{\prime}$, calculated for ${ }^{161} \mathrm{Hf}$ are shown in Fig. 6. The labeling convention for quasiparticles, is given in Table II. In these calculations, the pairing strength is calculated at zero frequency and is modeled to decrease with increasing rotational frequency such that the pairing has fallen by $50 \%$ at $\hbar \omega=0.70 \mathrm{MeV}$, as detailed in Ref. [16]. For deformation parameters we assume zero triaxiality and the parameters $\beta_{2}=0.161$ and $\beta_{4}=0.018$ were based on the predictions of Ref. [17].

In order to compare the experimental results with the calculations, the experimental alignments $i_{x}$ [18] are plotted in Fig. 7 as a function of rotational frequency. Also plotted is the yrast band in ${ }^{162} \mathrm{Hf}[4,20]$. A rotational reference, based on

TABLE II. Convention for the quasiparticle labeling.

\begin{tabular}{lcc}
\hline \hline Label & $\begin{array}{c}\text { Parity and signature } \\
(\pi, \alpha)\end{array}$ & $\begin{array}{c}\text { Main shell-model } \\
\text { component }\end{array}$ \\
\hline & Quasineutrons & \\
$\mathrm{A}$ & $(+,+1 / 2)_{1}$ & $i_{13 / 2}$ \\
$\mathrm{~B}$ & $(+,-1 / 2)_{1}$ & $i_{13 / 2}$ \\
$\mathrm{C}$ & $(+,+1 / 2)_{2}$ & $i_{13 / 2}$ \\
$\mathrm{E}$ & $(-,+1 / 2)_{1}$ & $h_{9 / 2}, f_{7 / 2}$ \\
$\mathrm{~F}$ & $(-,-1 / 2)_{1}$ & $h_{9 / 2}, f_{7 / 2}$ \\
& Quasiprotons & \\
$\mathrm{A}_{\mathrm{p}}$ & $(-,-1 / 2)_{1}$ & $h_{11 / 2}$ \\
$\mathrm{~B}_{\mathrm{p}}$ & $(-,+1 / 2)_{1}$ & $h_{11 / 2}$ \\
\hline \hline
\end{tabular}




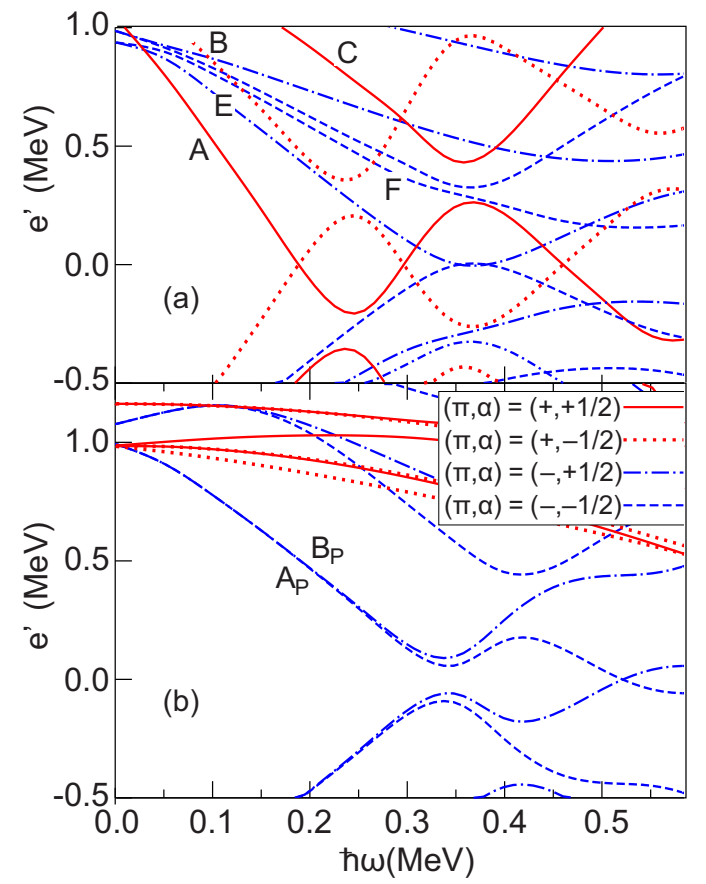

FIG. 6. (Color online) Cranked Woods-Saxon Routhian diagrams for (a) quasineutrons and (b) quasiprotons in ${ }^{161} \mathrm{Hf}$ using deformation parameters $\beta_{2}=0.161, \beta_{4}=0.018$, and $\gamma=0^{\circ}$. The quasiparticles are labeled with the convention as given in Table II.

a configuration with a variable moment of inertia defined by the Harris parameters [19] $J_{0}=12.5 \hbar^{2} \mathrm{MeV}^{-1}$ and $J_{1}=85$ $\hbar^{4} \mathrm{MeV}^{-3}$, has been subtracted [4].

The Woods-Saxon cranking calculations shown in Fig. 6 indicate that the $i_{13 / 2}$ quasineutron A becomes the lowest in energy at low rotational frequencies. The yrast band of ${ }^{161} \mathrm{Hf}$ carries an initial alignment of $\sim 5.5 \hbar$ (see Fig. 7), which is consistent with the slope of the A Routhian $\left(i_{x}=-d e^{\prime} / d \omega[18]\right)$ predicted by the cranking calculations. Therefore at low rotational frequency the yrast band is interpreted at the $i_{13 / 2}$ quasineutron configuration $\mathrm{A}$.

Bands 1 and 2 , have an initial alignment of $\sim 12 \hbar$ at $\hbar \omega=0.25 \mathrm{MeV}$, which indicates that these bands are based on three-quasiparticle configurations (see Fig. 7). Bands 1 and 2 are likely to be the FAB and EAB three-quasineutron configurations. Analogous bands are seen throughout the region (e.g., [1,2]). At a rotational frequency of $\hbar \omega \sim 0.40 \mathrm{MeV}$, Band 2 undergoes a gain in alignment of $\sim 6 \hbar$, which is interpreted as the first $h_{11 / 2}$ proton crossing. This crossing is also observed in the neighboring nucleus ${ }^{162} \mathrm{Hf}$ (see Fig. 7) $[4,20]$. Band 1 also shows an indication of this crossing at the top of the band.

As the rotational frequency is increased the yrast band of ${ }^{161} \mathrm{Hf}$ displays a gradual gain in alignment up to $\sim 0.4 \mathrm{MeV}$ of $\sim 5 \hbar$ [see Fig. 7(b)]. At this point a gain in alignment of $\sim 6 \hbar$ is observed that is similar to the first proton crossing seen in Band 2 and in ${ }^{162} \mathrm{Hf}$. In order to understand the overall alignment behavior in ${ }^{161} \mathrm{Hf}$ the systematics of alignment in the lower mass $N=89$ is shown in Fig. 7(c). In the more deformed $N=89$ nuclei ${ }^{157} \mathrm{Er}$ [1] and ${ }^{159} \mathrm{Yb}$ [2] the first crossing in the yrast band is caused by the almost simultaneous

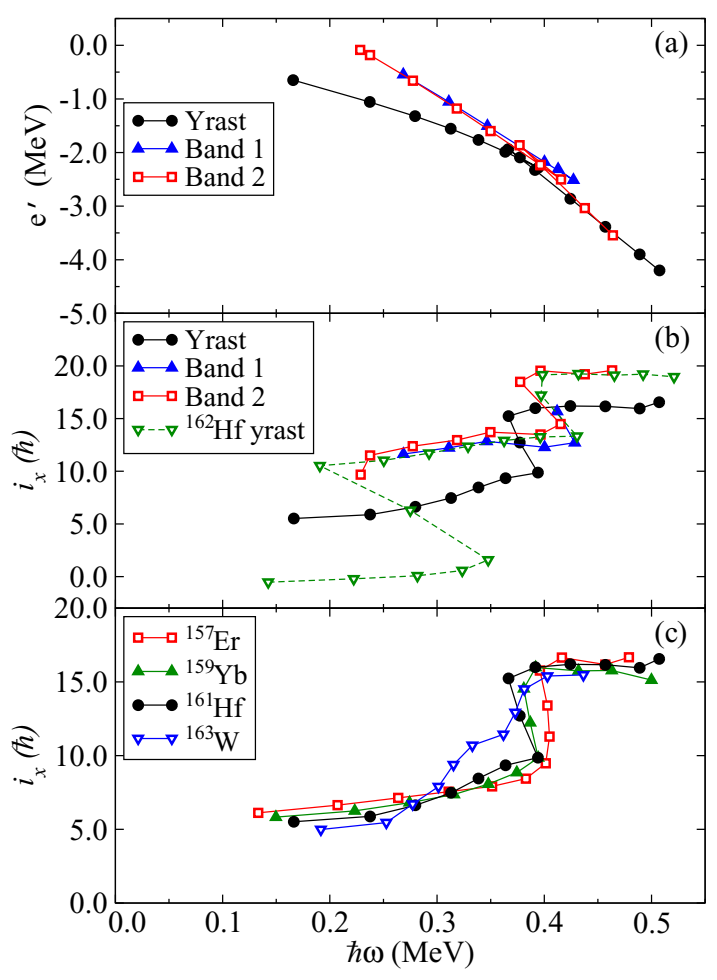

FIG. 7. (Color online) Experimental (a) Routhians, $e^{\prime}$, and (b) alignments, $i_{x}$, as a function of rotational frequency, $\hbar \omega$, for bands in ${ }^{161} \mathrm{Hf}$. Also shown in panel (b) is the alignment for the yrast band of ${ }^{162} \mathrm{Hf}[4,20]$. (c) Experimental alignments, $i_{x}$, for the yrast bands of odd-mass, $N=89$ isotones ${ }^{157} \operatorname{Er}[1],{ }^{159} \mathrm{Yb}$ [2], ${ }^{161} \mathrm{Hf}$, and ${ }^{163} \mathrm{~W}$ [3].

alignment of the second pair of $i_{13 / 2}$ quasineutrons $\mathrm{BC}$ and the first pair of $h_{11 / 2}$ quasiprotons $\mathrm{A}_{\mathrm{p}} \mathrm{B}_{\mathrm{p}}$. The overall gain in alignment in ${ }^{161} \mathrm{Hf}$ is similar to that observed in the lighter isotones and suggests that the full alignment involves these two pairs of quasiparticles. It is interesting to note that in the heavier and less deformed isotone ${ }^{163} \mathrm{~W}$ a double crossing is also observed; however, the first pair of neutrons to align are $\left(h_{9 / 2} / f_{7 / 2}\right) \mathrm{EF}$ rather than $\left(i_{13 / 2}\right) \mathrm{BC}$ [3]. The cranking calculations for quasineutrons in ${ }^{161} \mathrm{Hf}$ [Fig. 6(a)] predict that the $\mathrm{EF}\left(h_{9 / 2} / f_{7 / 2}\right)$ and the $\mathrm{BC}\left(i_{13 / 2}\right)$ neutrons align at roughly the same frequency, although the EF crossing has a strong interaction strength, which would result in a smooth alignment. Therefore, the additional alignment of the EF quasineutrons cannot be ruled out. The proposed configuration assignments and quasiparticle alignments that characterize the bands in ${ }^{161} \mathrm{Hf}$ are summarized in Table III.

TABLE III. Summary of configuration assignments for the bands in ${ }^{161} \mathrm{Hf}$.

\begin{tabular}{lcc}
\hline \hline Band & $(\pi, \alpha)$ & \multicolumn{1}{c}{$\begin{array}{c}\text { Quasiparticle configuration } \\
\text { assignment }\end{array}$} \\
\hline Yrast & $(+,+1 / 2)$ & $\mathrm{A} \rightarrow \mathrm{ABC} \rightarrow \mathrm{ABCA}_{\mathrm{p}} \mathrm{B}_{\mathrm{p}}$ \\
& or $\mathrm{A} \rightarrow \mathrm{ABCEF} \rightarrow \mathrm{ABCEFA}_{\mathrm{p}} \mathrm{B}_{\mathrm{p}}$ \\
Band 1 & $(-,-1 / 2)$ & $\mathrm{FAB} \rightarrow \mathrm{FABA}_{\mathrm{p}} \mathrm{B}_{\mathrm{p}}$ \\
Band 2 & $(-,+1 / 2)$ & $\mathrm{EAB} \rightarrow \mathrm{EABA}_{\mathrm{p}} \mathrm{B}_{\mathrm{p}}$ \\
\hline \hline
\end{tabular}




\section{B. Decay of the isomeric state}

A value for the $B(M 2)$ of $0.17(1)$ W.u. was obtained for the 202-keV transition using an $\alpha_{\text {tot }}$ taken from the BrIcc database [12] and assuming a $E 3 / M 2$ mixing ratio of $\delta=0$ and a $100 \%$ branching ratio. Such a value of $B(M 2)$ is typical for single-particle transitions between $i_{13 / 2}$ and $h_{9 / 2}$ transitions and a value of $0.18(4)$ W.u. seen in the neighboring odd $N=89$ isotone, ${ }^{163} \mathrm{~W}$ [21], for a transition of this type. Along with the alignment characteristics of the rotational band built on the isomer and the orbitals expected to be present at the Fermi surface in ${ }^{161} \mathrm{Hf}$, the $B(M 2)$ value indicates that the isomer is a $v i_{13 / 2}$ single-particle state. The $\left(9 / 2^{-}\right)$and $\left(7 / 2^{-}\right)$ states are likely to be principally based on the $v h_{9 / 2}$ and $v f_{7 / 2}$ single-particle states, respectively. It is probable that the $\left(7 / 2^{-}\right)$state is the ground state of ${ }^{161} \mathrm{Hf}$, as it is for the neighboring $N=89$ isotone, ${ }^{163} \mathrm{~W}$ [21].

\section{SUMMARY}

The level structure of the neutron-deficient nucleus ${ }^{161} \mathrm{Hf}$ has been established to high spin using the JUROGAM II and GREAT spectrometers in conjunction with the RITU gas-filled separator. Configuration assignments have been made based on the predictions of Wood-Saxon cranking calculations. The yrast band undergoes an alignment that is consistent with two crossings involving the second pair of $i_{13 / 2}$ quasineutrons BC and the first pair of $h_{11 / 2}$ quasiprotons $\mathrm{A}_{\mathrm{p}} \mathrm{B}_{\mathrm{p}}$, as observed in the lighter $N=89$ isotones. An alternative explanation is that the neutron crossing also involves the $\left(h_{9 / 2} / f_{7 / 2}\right)$ EF quasineutrons. These latter orbitals become energetically favored as the deformation decreases and are observed to align in the yrast band in the less deformed isotone ${ }^{163} \mathrm{~W}$. Two side bands are reported to high spin and in one band the $A_{p} B_{p}$ proton crossing is observed. The isomeric decay of the $\left(13 / 2^{+}\right)$bandhead of the yrast band is established and traced to a possible $\left(7 / 2^{-}\right)$ground state.

\section{ACKNOWLEDGMENTS}

Financial support for this work has been provided by the UK Science and Technology Facilities Council (STFC), the EU Seventh Framework Programme Integrating Activities, Transnational Access, Project No. 262010 (ENSAR), the Academy of Finland under the Finnish Centre of Excellence Programme 2012-2017, and the US National Science Foundation (Grant No. PHY-0754674). We thank the GAMMAPOOL European Spectroscopy Resource for the loan of detectors for JUROGAM II. The authors express their gratitude to the staff of the Accelerator Laboratory at the University of Jyväskylä for their excellent technical support. The authors also thank Paul Morrall of Daresbury Laboratory, John Greene of Argonne National Laboratory, and Raimo Seppälä from the University of Jyväskylä for preparation of the targets.
[1] J. Simpson et al., J. Phys. G: Nucl. Part. Phys. 15, 643 (1989).

[2] T. Byrski et al., Nucl. Phys. A 474, 193 (1987).

[3] J. Thomson et al., Phys. Rev. C 81, 014307 (2010).

[4] H. Hübel et al., Z. Phys. A 329, 289 (1988).

[5] C. W. Beausang and J. Simpson, J. Phys. G: Nucl. Part. Phys. 22, 527 (1996).

[6] M. Leino et al., Nucl. Instrum. Methods Phys. Res., Sect. B 99, 653 (1995).

[7] R. D. Page et al., Nucl. Instrum. Methods Phys. Res., Sect. B 204, 634 (2003).

[8] I. Lazarus et al., IEEE Trans. Nucl. Sci. 48, 567 (2001).

[9] P. Rahkila, Nucl. Instrum. Methods Phys. Res., Sect. A 595, 637 (2008).

[10] D. C. Radford, Nucl. Instrum. Methods Phys. Res., Sect. A 361, 297 (1995).

[11] D. M. Cullen et al., Phys. Rev. C 58, 846 (1998).
[12] T. Kibédi, T. W. Burrows, M. B. Trzhaskovskaya, P. M. Davidson, and C. W. Nestor, Jr., Nucl. Instrum. Methods A 589, 202 (2008).

[13] A. Krämer-Flecken et al., Nucl. Instrum. Methods Phys. Res., Sect. A 275, 333 (1989).

[14] W. Nazarewicz, J. Dudek, R. Bengtsson, and I. Ragnarsson, Nucl. Phys. A 435, 397 (1985).

[15] S. Cwiok, J. Dudek, W. Nazarewicz, K. Skalski, and T. Werner, Comput. Phys. Commun. 46, 379 (1987).

[16] R. Wyss, J. Nyberg, A. Johnson, R. Bengtsson, and W. Nazarewicz, Phys. Lett. B 215, 211 (1988).

[17] P. Möller, J. R. Nix, W. D. Myers, and W. J. Swiatecki, At. Data Nucl. Data Tables 59, 185 (1995).

[18] R. Bengtsson and S. Frauendorf, Nucl. Phys. A 327, 139 (1979).

[19] S. M. Harris, Phys. Rev. 138, B509 (1965).

[20] C. R. Bingham et al., J. Phys. G: Nucl. Phys. 14, L77 (1988).

[21] C. Scholey et al., Phys. Rev. C 81, 014306 (2010). 